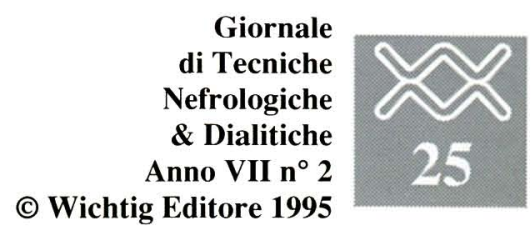

\title{
Anatomia del rene in ecotomografia
}

\author{
L. Pittavini, E. Nunzi, G. Quintaliani, L. Demegni, U. Buoncristiani \\ U.O. di Nefrologia e Dialisi - Ospedale Silvestrini - Perugia
}

n nefrologia l'ecografia dei reni ha trovato numerose applicazioni.

Per lo studio ecografico del rene si utilizzano apparecchiature in real time dotate di sonde preferenzialmente convex o settoriali con frequenze di $3.5 \mathrm{MHz}$.

Nei pazienti magri, nel bambino e nel rene trapiantato si ottengono immagini migliori utilizzando frequenze più elevate, preferibilmente di $5 \mathrm{MHz}$.

Le vie di accesso al rene sono:

- anteriore (addominale)

- laterale (lombare)

- posteriore (dorsale), oggi poco utilizzata, eccetto quale approccio per la biopsia renale o nel neonato in cui il meteorismo addominale ostacolerebbe la visualizzazione.

La conoscenza dell'anatomia della loggia renale e di tutti i suoi rapporti, dell'area pelvica e delle anomalie che si possono congenitamente verificare (distopie, anomalie di forma, di numero...), permetterà una adeguata interpretazione dell'immagine ecografica, il tutto tenendo conto della fisica degli ultrasuoni.

Numerose finestre acustiche e lo sfruttamento di opportuni decubiti consentono di ottenere immagini soddisfacenti nella maggior parte dei casi.

Le scansioni vanno eseguite tutte, per essere sicuri di aver esplorato l'organo nella sua interezza completate da eventuali scansioni oblique adatte alle situa- zioni che si possono presentare in base alla conformazione fisica del soggetto (Tab. I).

Per ridurre l'entità dell'ostacolo costituito dall'arcata costale si ricorre alla collaborazione del paziente cui viene chiesto di trattenere il respiro in inspirazione forzata; questo permette di sfruttare meglio la finestra acustica epatica. Anche il decubito laterale favorisce la visualizzazione a destra ed è addirittura praticamente indispensabile a sinistra perché riduce l'interferenza dell'aria colica.

Per studiare ecograficamente il rene sinistro è spesso necessario far assumere al paziente il decubito laterale, inserendo un cuscino sotto il fianco destro per aumentare lo spazio tra coste e ala iliaca sinistra. (Figg. 1, 13).

L'immagine in real time dovrebbe essere analizzata ed elaborata mentalmente dall'operatore in maniera da poter ricavare quante più informazioni possibile e in particolare:

- Sede e posizione

- Mobilità

- Forma e profilo esterno

- Dimensioni

- Parenchima

- Seno renale

- Ilo renale (vasi, complesso pielocaliciale)

- Via escretrice compresa la vescica

- Rapporti con organi e strutture circostanti.

\section{Sede, posizione, mobilità}

Il rene va ricercato per prima cosa nella sua sede naturale cioè nella loggia lombare. Se in tale sede non è riconoscibile, può trattarsi di una agenesia $o$, più spesso, di una ectopia, per cui l'esplorazione ecografica deve essere estesa a qualsiasi punto sul percorso di migrazione embriologica tra la sede normale e la vescica e anche contralateralmente (difetti congeniti di migrazione).

Ecograficamente è valutabile l'inclinazione del rene sui piani frontale e sagittale nonché la rotazione sull'asse longitudinale dell'organo.

L'inclinazione normale del rene sul piano sagittale è mediamente nell'uomo di $28,40^{\circ}$ e nella donna di $29,50^{\circ}$.

Alcuni Autori utilizzano il diaframma

TAB. I - SCANSIONI ECOGRAFICHE PER LO STUDIO DEL RENE

- Scansione longitudinale anteriore

- Scansione assiale anteriore

- Scansione longitudinale laterale (coronale)

- Scansione assiale laterale

- Scansione longitudinale obliqua posteriore

- Scansione longitudinale posteriore

- Scansione assiale posteriore 

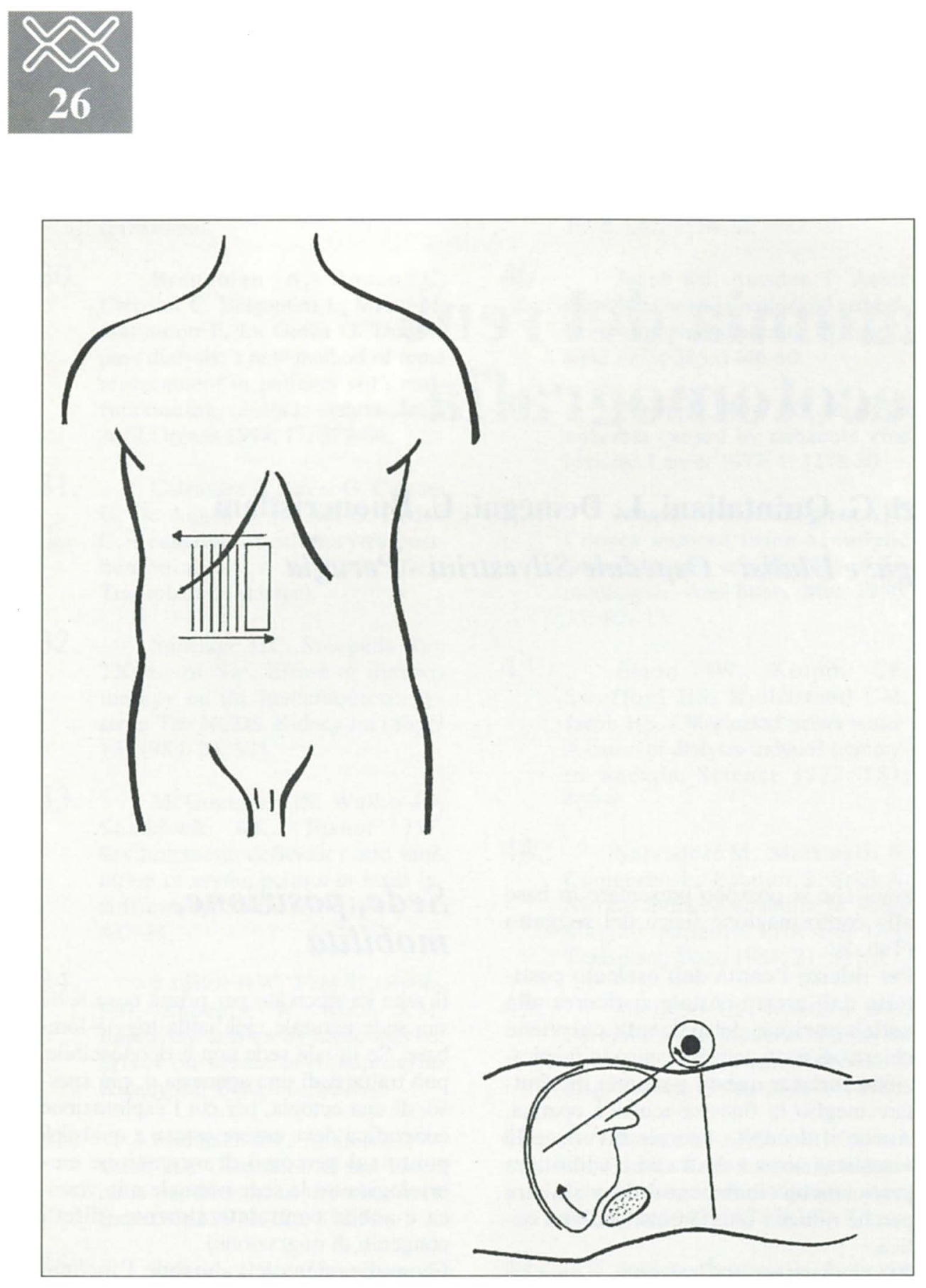

Fig. 1 - Esplorazione del rene in scansione longitudinale anteriore.

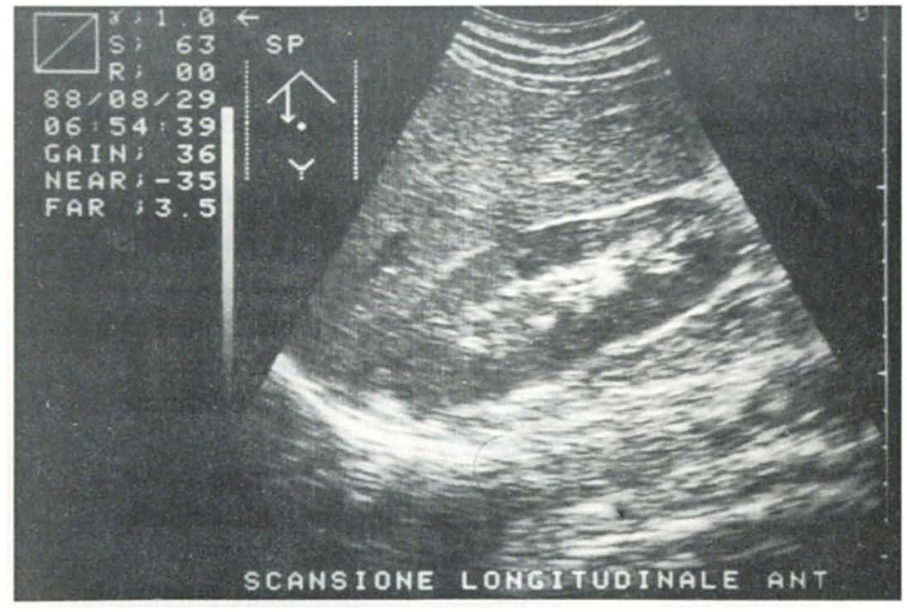

Fig. 2 - Rene destro in scansione longitudinale anteriore.

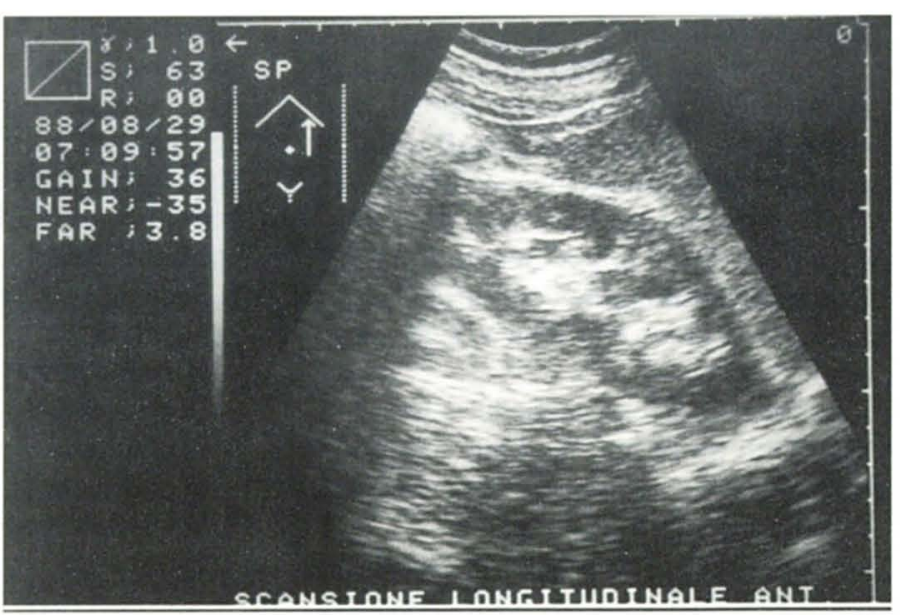

Fig. 3 - Rene sinistro in scansione longitudinale anteriore. 


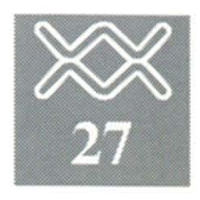

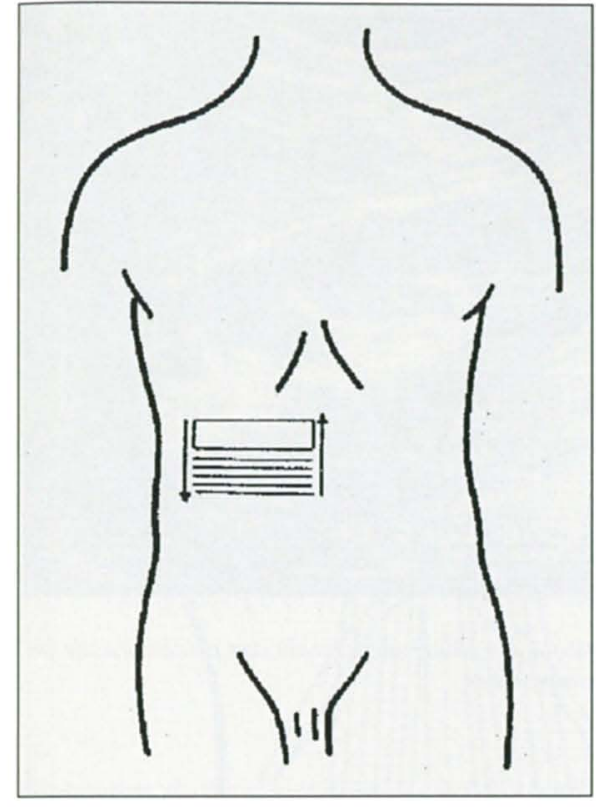

Fig. 4 - Esplorazione del rene in scansione assiale anteriore.

Fig. 6 - Esplorazione del rene in scansione longitudinale laterale o coronale.
Fig. 5 - Rene destro in scansione assiale anteriore.
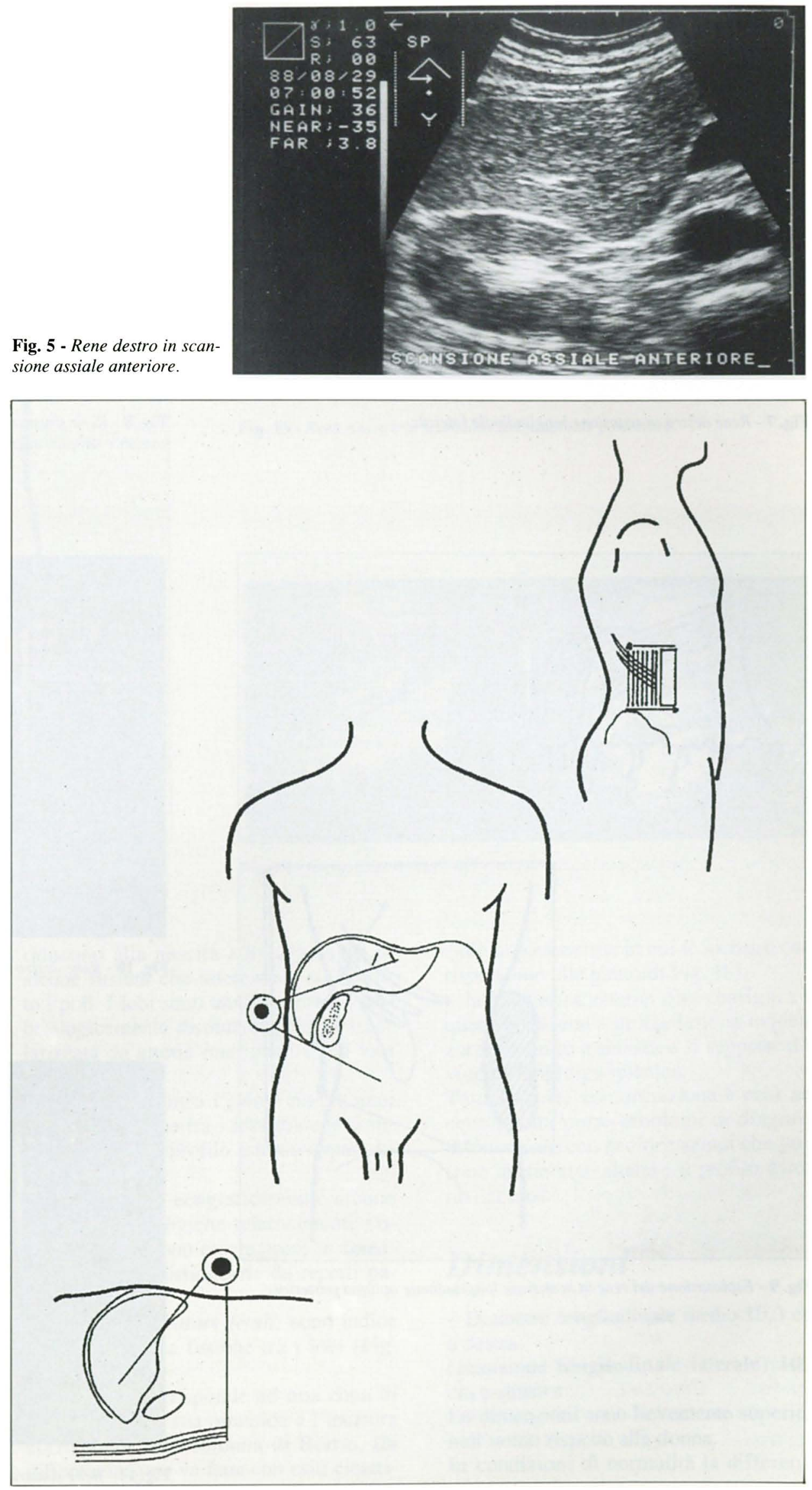


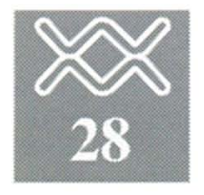

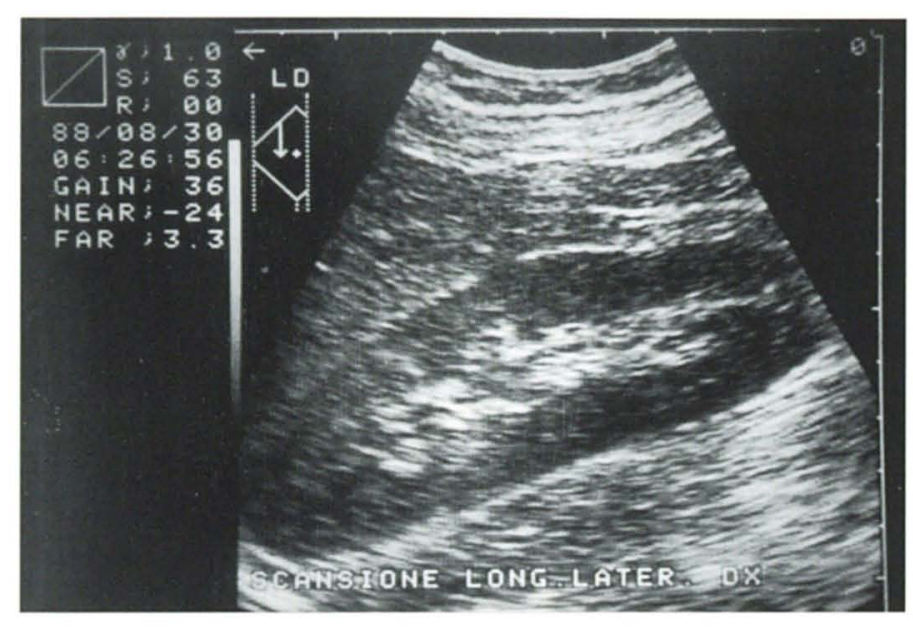

Fig. 7 - Rene destro in scansione longitudinale laterale.

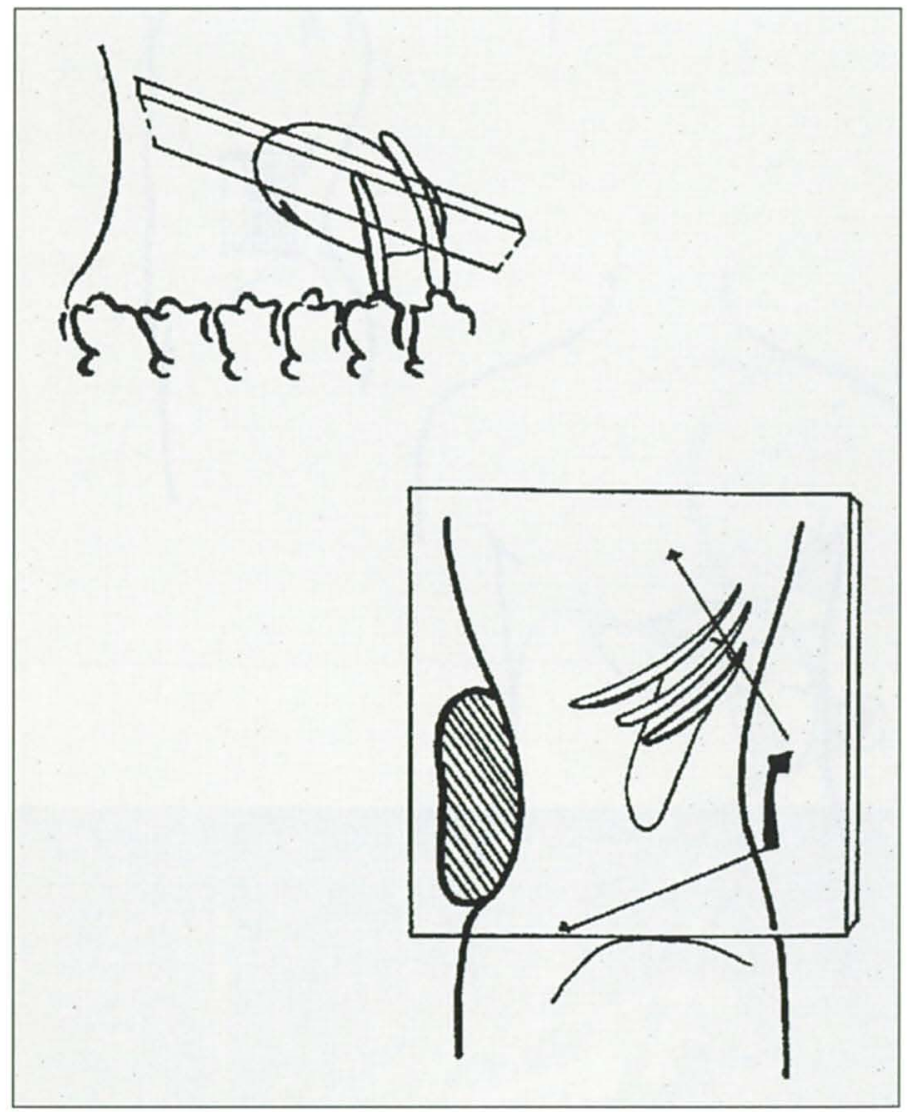

Fig. 9 - Esplorazione del rene in scansione longitudinale obliqua posteriore.

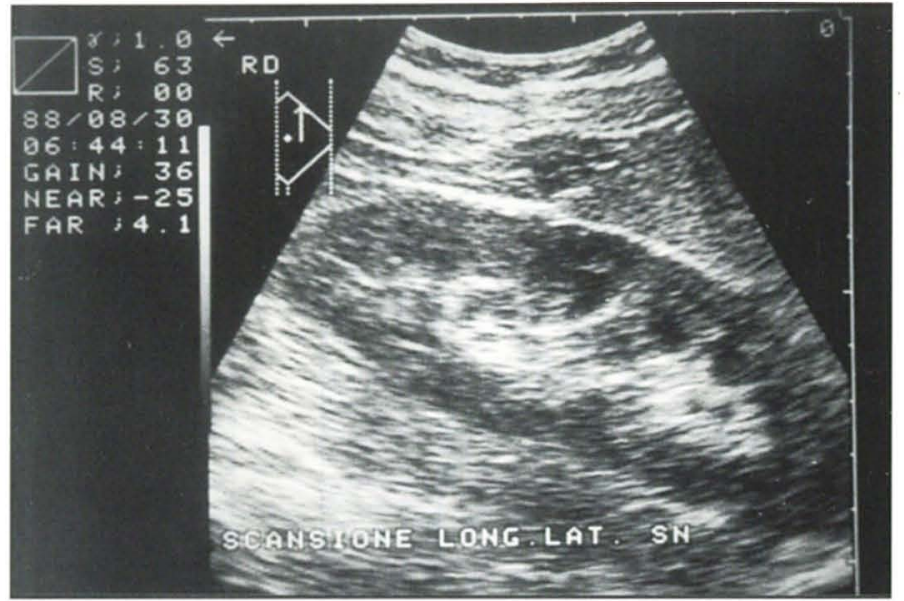

Fig. 8 - Rene sinistro in scansione longitudinale laterale con evidenza delle piramidi e del passaggio corticomidollare.

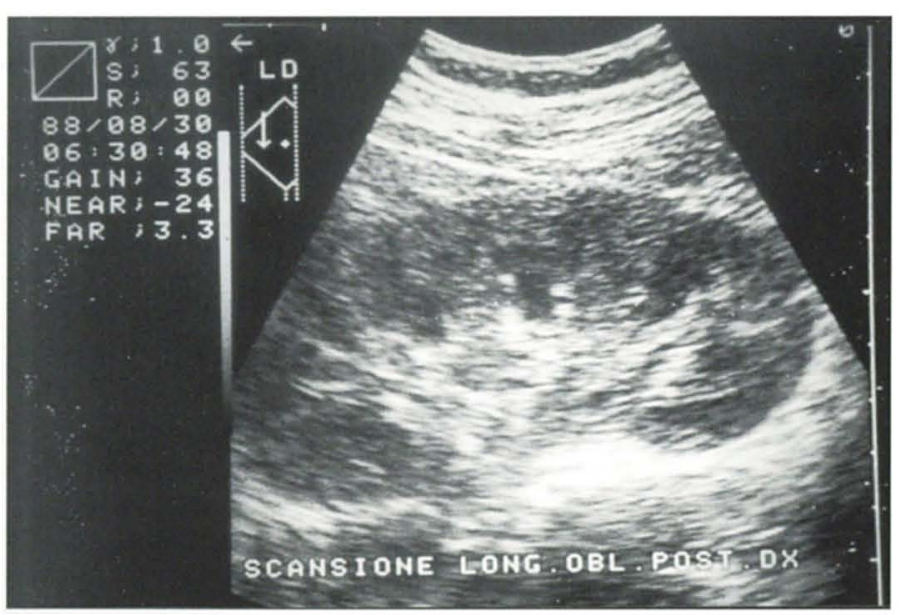

Fig. 10 - Rene destro in scansione longitudinale obliqua posteriore.

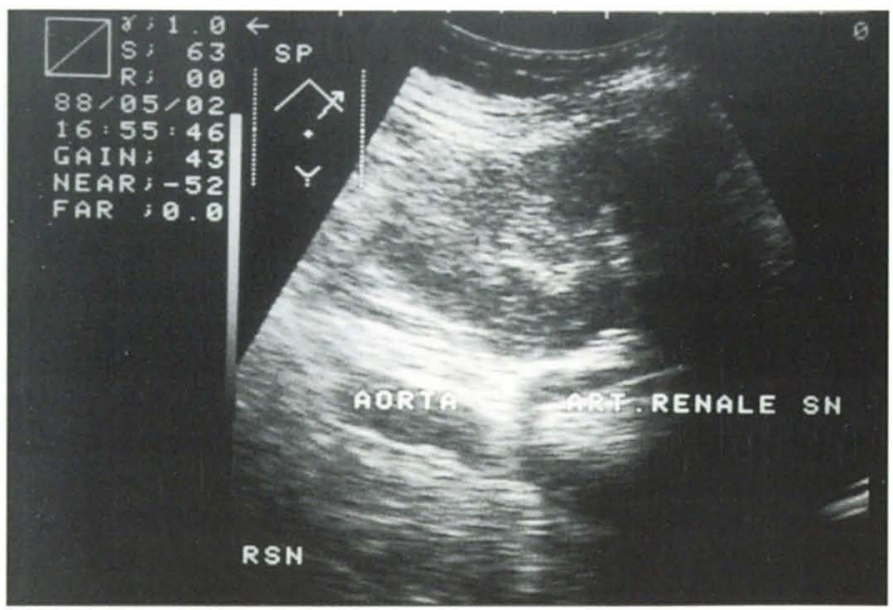

Fig. 11 - Rene sinistro in scansione longitudinale obliqua posteriore. 


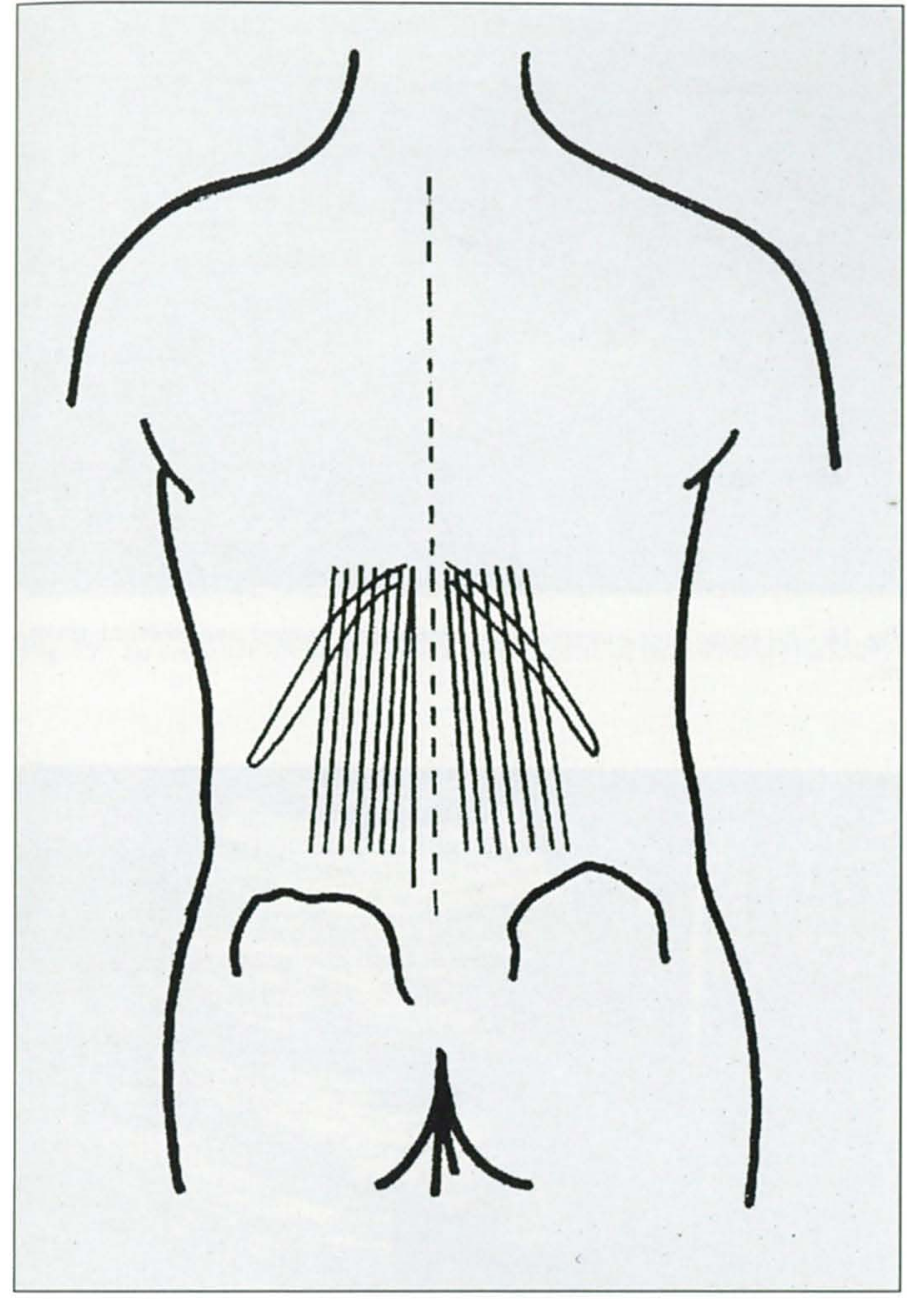

Fig. 12 - Esplorazione del rene in scansione longitudinale posteriore.

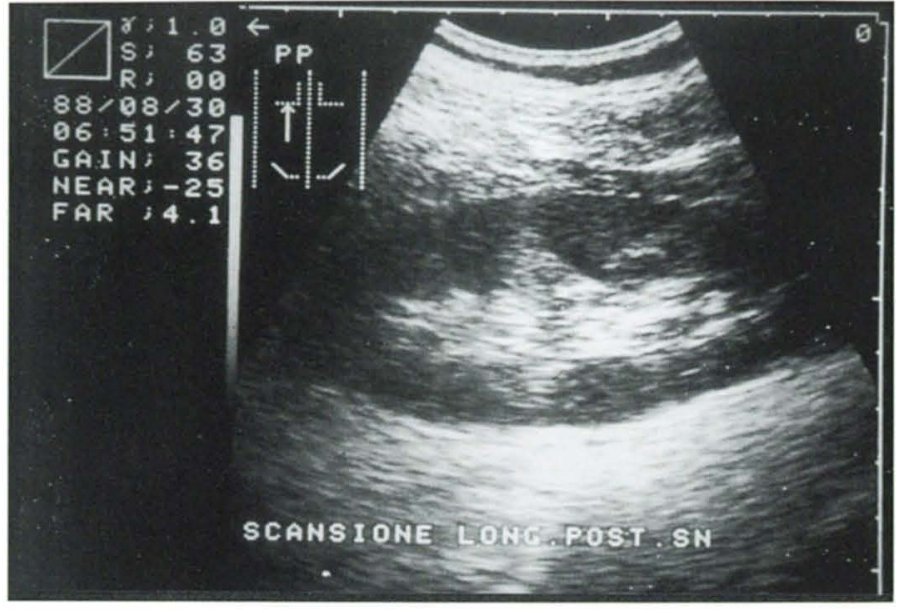

Fig. 13 - Rene sinistro in scansione longitudinale posteriore.

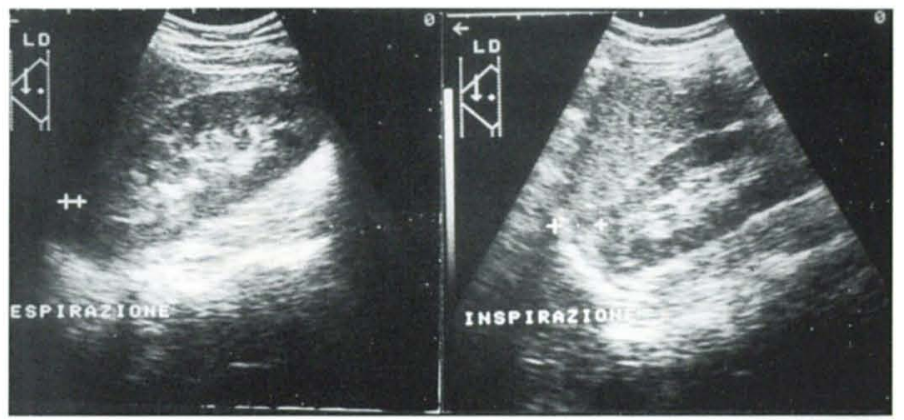

Fig. 14 - Escursione normale del rene con gli atti respiratori. come piano di riferimento dal quale misurare la distanza del polo superiore del rene durante l'apnea inspiratoria. Il rene si considera in posizione normale per una distanza massima del polo superiore dal diaframma di $4 \mathrm{~cm}$, dopo di che si parla di ptosi renale.

Le escursioni del rene legate agli atti respiratori in condizioni normali sono comprese tra i 2 e i $3.5 \mathrm{~cm}$ (Fig. 14).

La mobilità legata alla variazione di decubito deve essere ricercata eseguendo l'esame ecografico, nelle stesse condizioni respiratorie prima in decubito supino e poi in ortostatismo nel sospetto di un rene mobile (Fig. 15 e 16).

\section{Profilo esterno}

Il rene è costituito embriologicamente da 14 lobi ( 7 anteriori e 7 posteriori) che si riducono alla nascita a $8-12$ a causa di alcune fusioni che interessano soprattutto i poli. I lobi sono unità funzionali embriologicamente distinte ciascuna vascolarizzata da arterie interlobari e dai vasi arciformi.

Normalmente entro i primi cinque anni di vita la fusione tra i lobi diviene completa per cui il profilo esterno appare liscio.

Si riconoscono ecograficamente alcune varianti morfologiche relativamente comuni che debbono essere prese in considerazione per distinguerle da reperti patologici:

- Incisure e lobature fetali: sono indice della incompleta fusione tra i lobi (Fig. 17).

La lobatura corrisponde ad una zona di corticale con la sua piramide e l'incisura corrisponde alla colonna di Bertin. (la differenziazione va fatta con esiti cicatri- ziali da pielonefrite in cui le incisure corrispondono alle piramidi Fig. 18).

- la bozzatura esterna che configura il quadro del rene a dromedario si evidenzia soprattutto a sinistra e si suppone dovuto all'impronta splenica.

Talora questa conformazione è così accentuata da porre problemi di diagnosi differenziale con neoformazioni che possono anche esse alterare il profilo esterno del rene.

\section{Dimensioni}

- Diametro longitudinale medio $10,3 \mathrm{~cm}$ a destra

(scansione longitudinale laterale) 10,4 cm a sinistra

Le dimensioni sono lievemente superiori nell'uomo rispetto alla donna.

In condizioni di normalità la differenza 


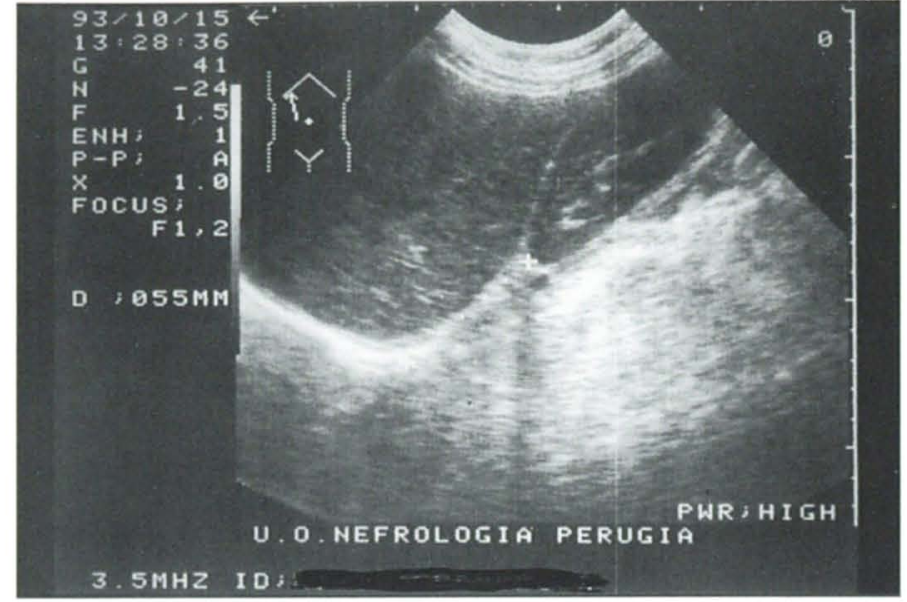

Fig. 15 - Rene destro esaminato in clinostatismo.

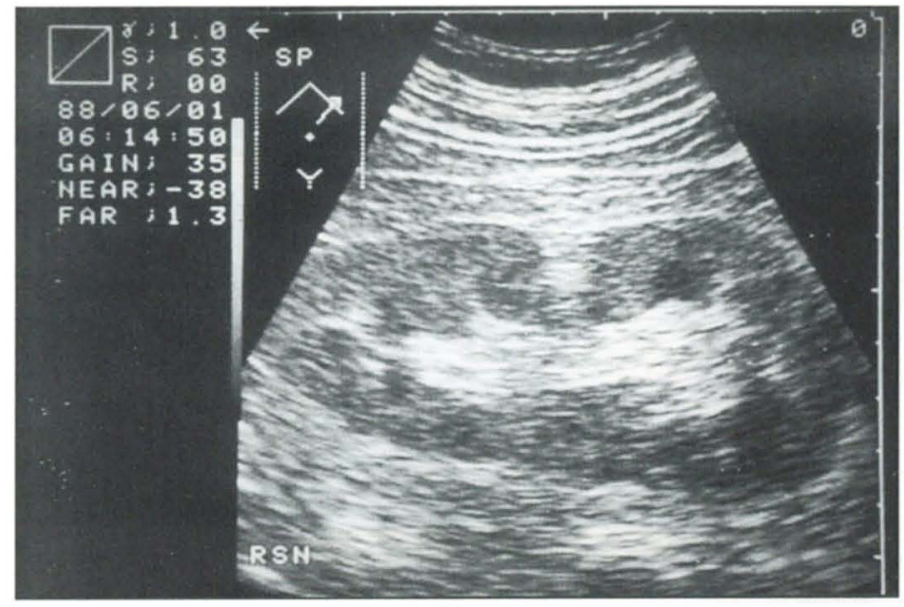

Fig. 17 - Evidente lobatura fetale per incompleta fusione.

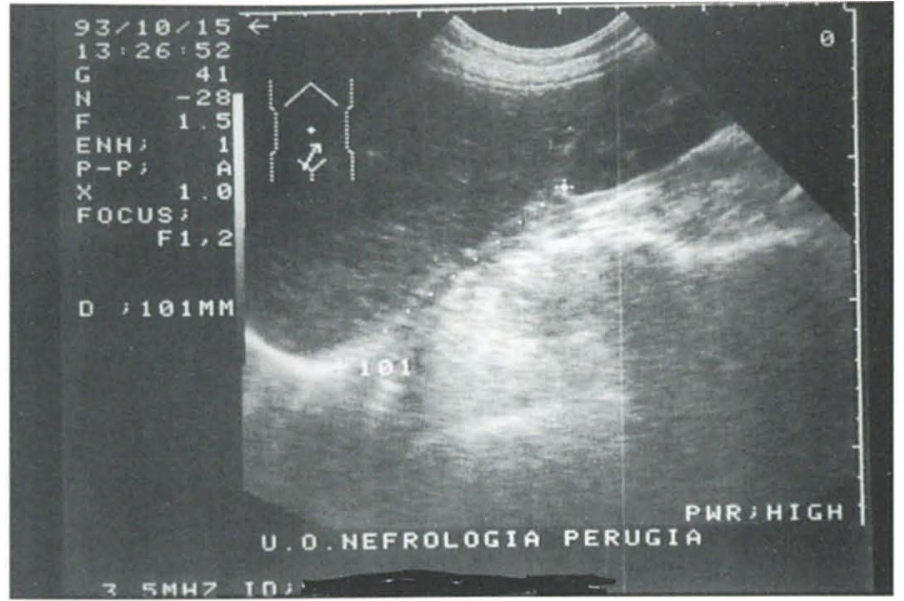

Fig. 16 - Lo stesso rene esaminato in ortostatismo mostra una mobilità abnorme.

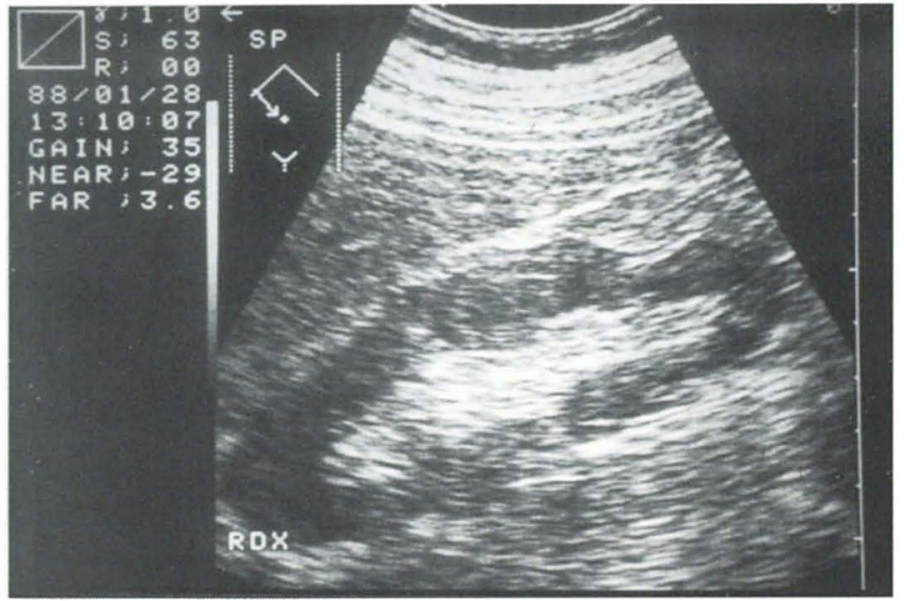

Fig. 18 - Cicatrice pielonefritica: la profonda incisura $\grave{e}$ in corrispondenza di una piramide. È presente anche retrazione della capsula e del grasso perirenale. del diametro longitudinale tra i due reni non supera i $15 \mathrm{~mm}$.

Le dimensioni ecografiche del rene sono del $15-30 \%$ inferiori rispetto a quelle radiologiche (ingrandimento radiografico, introduzione del m.d.c.).

La grandezza dei due reni aumenta con l'età fino ai 30 anni, poi si riduce lentamente, dopo una fase stazionaria (G. Rizzato e coll. 1987).

\section{Parenchima}

Il parenchima in condizioni normali è nettamente distinto dal seno renale e in esso si riconoscono la corticale e la midollare.

La corticale è rappresentata da echi di li- vello medio basso, uniformemente distribuiti, è più ecogena della midollare, probabilmente per il maggior numero di interfacce dovuto alla ricchezza di vasi.

La midollare comprende aree ipoecogene grossolanamente triangolari (le piramidi) separate da corticale (colonne di Bertin).

La minore ecogenicità della midollare rispetto alla corticale è legata al maggior contenuto idrico di questa e alla disposizione parallela del fascio ultrasonoro rispetto alle strutture tubulari.

La superficie esterna del parenchima è liscia e regolare delimitata dalla ecogenicità della capsula.

La superficie interna, rivolta verso il seno, è frastagliata per la presenza delle piramidi e colonne di Bertin.
In ecografia nefrologica è considerata di fondamentale importanza la valutazione della ecogenicità della corticale. Essa viene messa a confronto con il parenchima epatico normale e si parlerà rispetto a quello di:

\section{ipoecogeno \\ isoecogeno \\ iperecogeno}

Prima di esprimersi sul grado di ecogenicità bisogna porre estrema attenzione per escludere la possibilità che sia solo il risultato di un artefatto, legato ad eccessiva amplificazione, ad una focalizzazione non corretta, alla interposizione di una falda liquida o di una colecisti distesa, oppure che l'organo di riferimento non 


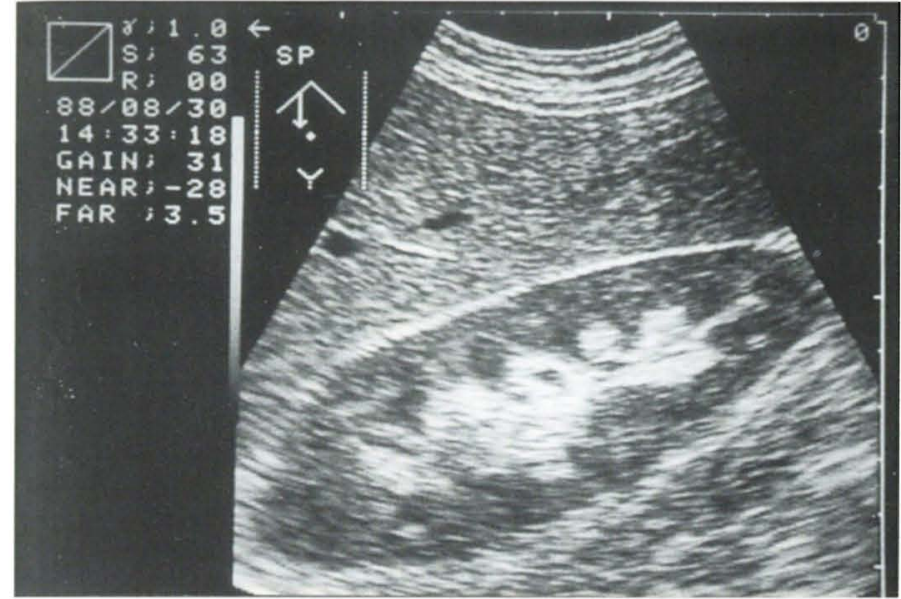

Fig. 19 - La corticale normale è ipoecognea rispetto al parenchima epatico.

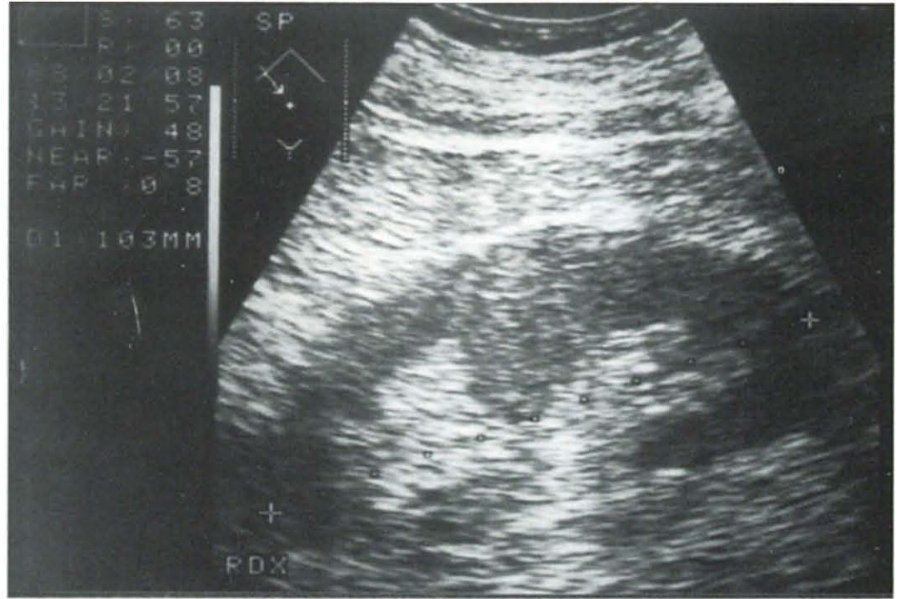

Fig. 20 - Colonna di Bertin ipertrofica. sia esso stesso sede di patologia che ne alteri l'ecogenicità.

Come in tutte le applicazioni, l'esperienza deve permettere all'operatore di non incorrere in queste errate valutazioni.

Si è cercato di trovare correlazioni tra l'ecogenicità corticale e la condizione funzionale renale.

In età neonatale e pediatrica l'ecogenicità della corticale tende ad essere sovrapponibile a quella del fegato, mentre in età adulta è inferiore a quella dell'organo di confronto. Una accentuazione della ipoecogenicità potrà essere rilevata in corso di affezioni acute nelle quali si verifica un edema dell'organo e quindi una interposizione di una maggiore quantità di liquido tra le interfacce.

In questi casi solitamente è aumentato anche lo spessore del mantello corticale e l'organo in toto. Anche la distribuzione della ecogenicità può risultare non più uniforme, ma a zolle.

L'incremento delle ecogenicità è nella maggior parte dei casi il risultato di una condizione patologica sia acuta che cronica, per lo più accompagnata da una modificazione dello spessore.

La ricerca del grado di definizione del passaggio corticomidollare è oggetto di numerose speculazioni.

La base piramidale, utilizzando opportune scansioni, (assiali anteriori, sagittali anteriori o coronali) risulta separata dalla corticale da echi lineari di elevata intensità determinati dai vasi arciformi.

Questi reperti e, in generale, la distinzione corticomidollare sono agevolmente visibili nel rene neonatale, nel rene pediatrico e nel rene trapiantato, mentre anche, in condizioni normali, possono non essere evidenziabili nel rene normale dell'adulto.

La mancata differenziazione corticomidollare non ha di per sè significato patologico e anzi in talune affezioni può essere conservata o addirittura accentuata (Fig. 19).

\section{Seno renale (zona pielovascolare centrale)}

È una zona iperecogena ovalare o rotondeggiante, a seconda delle scansioni, compresa tra il profilo frastagliato del parenchima e l'ilo renale.

L'elevata ecogenicità è dovuta alla presenza di numerose interfacce e componenti a diversa impedenza acustica (sistema collettore, vasi, nervi, linfatici, tessuto fibroso, grasso).

Solitamente l'immagine ecografica del seno renale è continua, ma può risultare interrotta parzialmente per una colonna di Bertin ipertrofica (per lo più mesorenale) o totalmente quando un istmo di parenchima divide in due il seno, andando ad unirsi al parenchima contrapposto come nel doppio distretto renale (Fig. 20).

La lipomatosi del seno costituisce una variante ecostrutturale del seno renale presentandosi come aree ipo-anecogene, prive di una parete ben definita, senza rinforzo posteriore di parete (come nelle cisti). Quando è particolarmente diffusa può creare problemi di diagnosi differenziale con le cisti renali parapieliche o addirittura con una calicopielectasia.

\section{Ilo renale}

È una struttura posta in continuità con il seno renale (di cui mantiene l'ecogenicità).

È delimitato dai labbri renali (nelle scansioni assiali) e dai tubercoli renali (nelle scansioni longitudinali oblique posteriori). A volte i tubercoli renali possono essere ipertrofici tali da porre problemi di diagnosi differenziale con neoformazioni.

La visualizzazione dell'ilo renale sinistro non è sempre possibile per l'inteferenza del meteorismo intestinale.

\section{Vasi renali}

Nelle scansioni assiali soprattutto a destra, si evidenziano strutture lineari, con parete ben distinguibile riferibili all'arteria e alla vena renale, di cui è abbastanza facilmente dimostrabile la continuità con l'aorta e la vena cava rispettivamente. A sinistra il meteorismo intestinale spesso ostacola la loro visualizzazione. Normalmente la vena è posta su un piano anteriore rispetto all'arteria. Il bacinetto è posto posteriormente alle strutture vascolari (Fig. 21). 


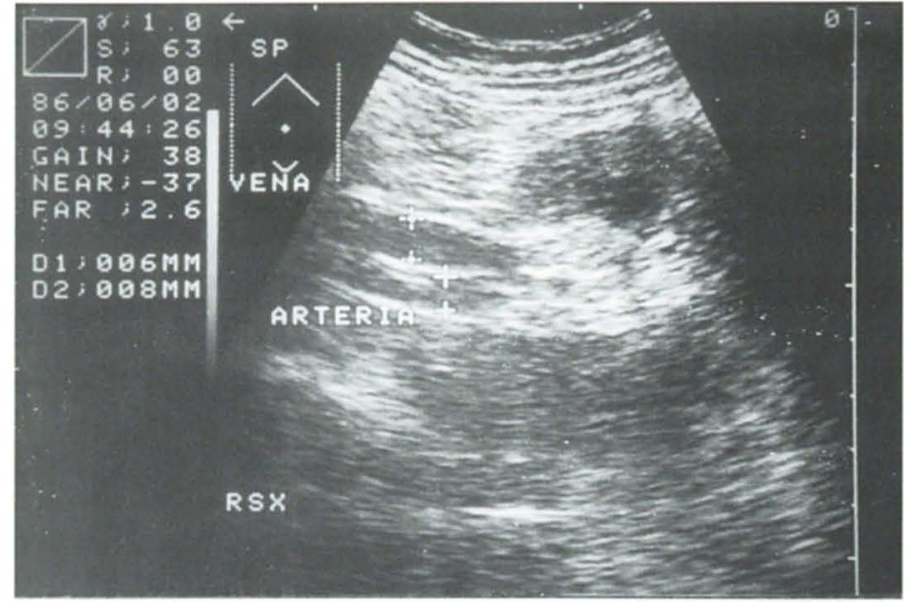

Fig. 21 - Peduncolo vascolare del rene sinistro.

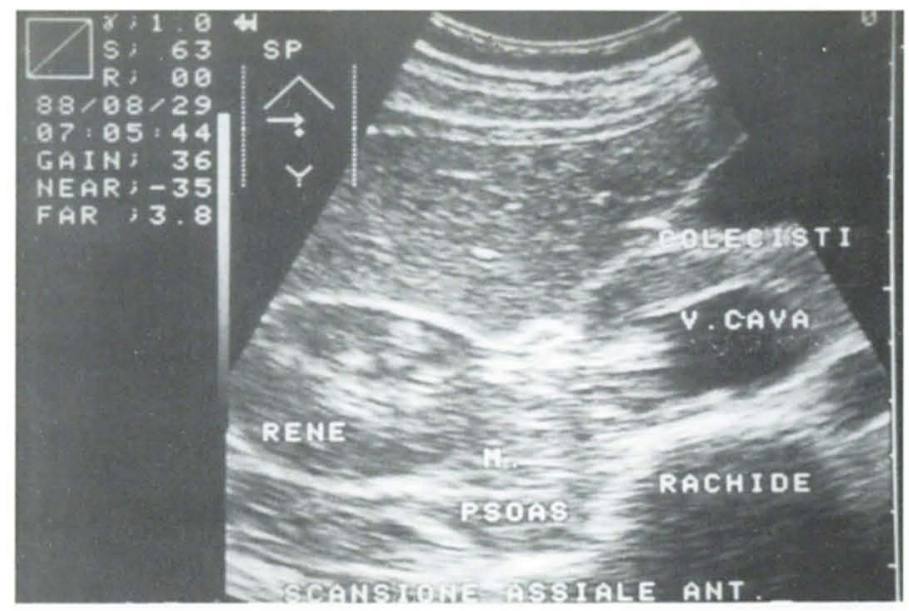

Fig. 23 - Rapporti anatomici del rene destro in scansione assiale anteriore.

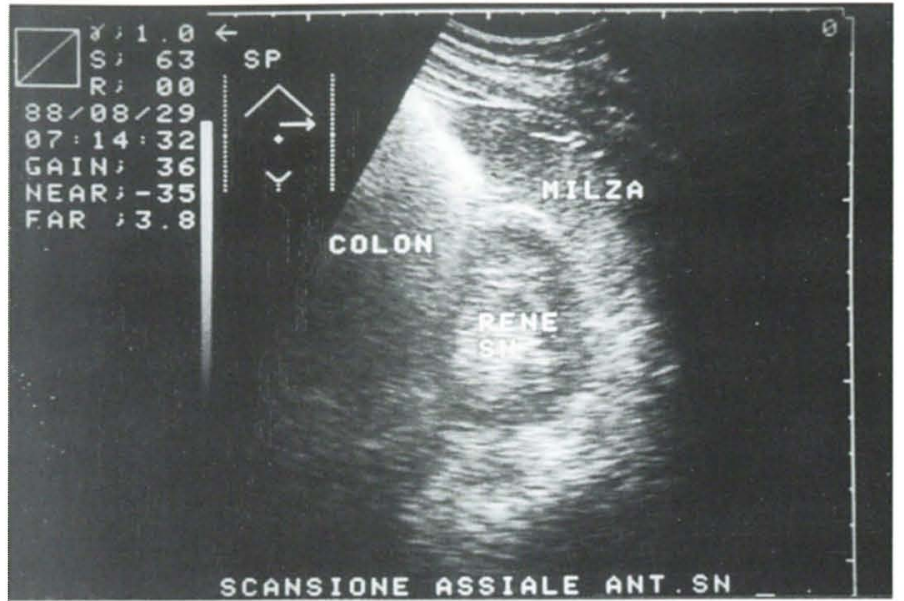

Fig. 22 - Rapporti anatomici del rene sinistro in scansione assiale anteriore.

TAB. II - RAPPORTI ANATOMICI DEL RENE SINISTRO

- Parete posteriore dello stomaco

- Colon trasverso

- Flessura splenica del colon

- Coda del pancreas

- Milza

- Ultimi archi costali

- Arcata lombo-costale e quadrato dei lombi

- Muscolo psoas

- Processi trasversi del rachide lombo-dorsale

- Aorta addominale

- Surrene sinistro

\section{Via escretrice (calici, bacinetto, uretere e vescica)}

Lo studio completo dell'anatomia renale non può non essere completato con quello della via escretrice, per le implicazioni che patologie di quest'ultima hanno sulla funzione e sulla struttura stessa del rene.

Calici e bacinetto vanno ricercati con scansioni coronali e longitudinali oblique posteriori. In condizioni di idratazione normale possono non essere dimostrabili.

In condizioni di iperidratazione o di carico diuretico o con la vescica notevolmente distesa (per retrostasi) è possibile osservare immagini ipoanecogene ramificate (i calici), che confluiscono verso una struttura anecogena centrale (la pelvi). In queste situazioni si può studiare la giunzione pieloureterale e talora i primissimi $\mathrm{cm}$ dell'uretere prossimale. L'uretere torna ad essere visualizzabile nei tratti iuxtavescicale e intramurale in condizioni di medio riempimento vescicale con scansioni sovrapubiche opportunamente angolate. L'uretere può essere cercato all'incrocio con i vasi iliaci e sarà visibile se è dilatato.

\section{Rapporti anatomici con organi e strutture circostanti}

Studiando l'anatomia ecografica dei reni si deve tenere conto dei rapporti anatomici che contrae perché patologie a carico dei reni possono interessare e
TAB. III - RAPPORTI ANATOMICI DEL RENE DESTRO

- Faccia posteroinferiore del lobo destro del fegato

- Colecisti

- Seconda porzione del dueodeno

- Flessura epatica del colon

- Ultimi archi costali

- Arcata lombocostale e del muscolo quadrato dei lombi

- Muscolo psoas

- Processi trasversi del rachide dorsolombare

- Vena cava

- Surrene destro

coinvolgere anche gli organi circostanti e viceversa (Tab. II, III). 


\section{Conclusione}

L'avvento dell'ecografia renale ha completamente modificato l'approccio diagnostico del paziente che accede al Reparto nefrologico, soppiantando in molte occasioni la radiodiagnostica tradizionale.

Il Nefrologo ecografista può integrare le sue conoscenze cliniche con le immagini avendo spesso indicazioni per la diagnosi, per la prognosi e per l'approccio terapeutico.

La biopsia renale da sempre complemento insostituibile della diagnostica per il Nefrologo, ha trovato nell'ecografia una guida pressoché indispensabile sia per la sua esecuzione che per il monitoraggio successivo, permettendo un prelievo idoneo nella maggior parte dei casi e un intervento tempestivo sulle eventuali complicanze.

\section{BIBLIOGRAFIA}

1.

Albarelli JN, Lawson TL. Renal ultrasonography advantages of the decubitus position. J Clin Ultrasound 1978; 6: 115-6.

2. Bazzocchi M, Rizzatto G. The value of the posterior oblique longitudinal scan in renal ultrasonography. Urol Radiol 1980; 1: 221.

3. Matter D. Ecografia dell'apparato urinario. Masson, Milano 1988.

4. Rabassini A. Rene: metodica e anatomia ecografica. In: Ecografia dell'apparato urogenitale. Torino: Ed. Minerva Medica 1992; 57-65.

5. Rizzatto G, Bazzocchi M. Reni in anatomia ecografica ed. Masson 1990.

6. Rosi P. Uretere normale e patologico. In: Ecografia dell'apparato urogenitale. Torino: Ed. Minerva Medica, 1992; 139-44.

7. Ziviello M, Biggi E, et al. Ecotomografia. Idelson, Napoli 1986. 